\title{
A Case Report of a Soft Lesion on The Buttock: A Classical Form of Naevus Lipomatosis Superficialis in a Kurdish Adult
}

\author{
Ebtisam Elghblawi
}

SJH, Tripoli, Libya

Correspondence should be addressed to: Ebtisam Elghblawi; ebtisamya@yahoo.com

Received date: 22 April 2014; Accepted date: 11 August 2014; Published date: 29 January 2015

Academic Editor: Mustafa Turhan Şahin

Copyright (C) 2015. Ebtisam Elghblawi. Distributed under Creative Commons CC-BY 4.0

\begin{abstract}
Naevus lipomatosis cutaneous superficialis (NLCS) is a rare benign hamartoma of adipose tissue of the skin characterized by the presence of clusters of mature ectopic fat cells among the collagen bundles of the dermis. Usually, the number of adnexal structures is reduced in NLCS with preserved morphology. NLCS is also recognized as "Naevus lipomatosis of Hoffman and Zurhelle". NLCS is presented as a group of soft ivory-skin coloured nodules mostly on the lower back, buttock, and abdomen. The nodules can be sessile and single, or grouped and pedunculated with smooth, wrinkled surface. They could present early in life or develop later between the second and sixth decade. The histopathological structure illustrates strands of mature fat cells in the dermis with intersecting collagen bundles. I am presenting a rare case of adult-onset classical NLCS, where the patient was emotionally distressed, depressed and mentally unstable as a result of its physical appearance.
\end{abstract}

Keywords: Naevus lipomatosis Superficialis, ipsilateral, nodules.

\section{Introduction}

Naevus lipomatosis superficialis is a rare idiopathic abnormality characterized by isolated dermal collection of adipose tissue as Ghosh et al (2010), Bancalari et al (2011), Varadraj et al (2013), Pujani et al (2014) defined.

Clinically, there are two forms of presentations. The classical form which was described first - by Hoffman and Zurhelle, is characterized by groups of multiple, nontender, soft, pedunculated, cerebriform, yellowish or skin-colored papules, nodules, or plaques. They are most commonly found over the lower back, buttocks and hips as Buch et al (2005), Das et al (2006) and Yap (2009) stated. Usually they are present from birth (congenital). The second form of NLCS clinically manifests itself as a solitary domeshaped or sessile papule mimicking skin tags

Cite this Article as: Ebtisam Elghblawi (2015)," A Case Report of a Soft Lesion on The Buttock: A Classical Form of Naevus Lipomatosis Superficialis In A Kurdish Adult ", JMED Research, Vol. 2015 (2015), 
developing mostly in adults and has been reported to occur over several other sites apart from the lower back. These areas include the arms, knees, axillae, ears, eyelids, and scalp as Khandpu et al, 2009 acknowledged.

\section{Case Presentation}

An otherwise healthy 41-year-old Iraqi Kurdish man presented with a large ivoryskin coloured plaque, approximately $5 \times 1.5$ $\mathrm{cm}$, consisting of irregular surfaced multiple soft papules, and nodules with irregular distinctive borders extending over the lower part of his right gluteal area in a linear, or zosteriform but not crossing the midline (segmental) distribution. The patient stated it had been present for 10 years.

On examination, lesions were unilateral in distribution and were soft and painless. They

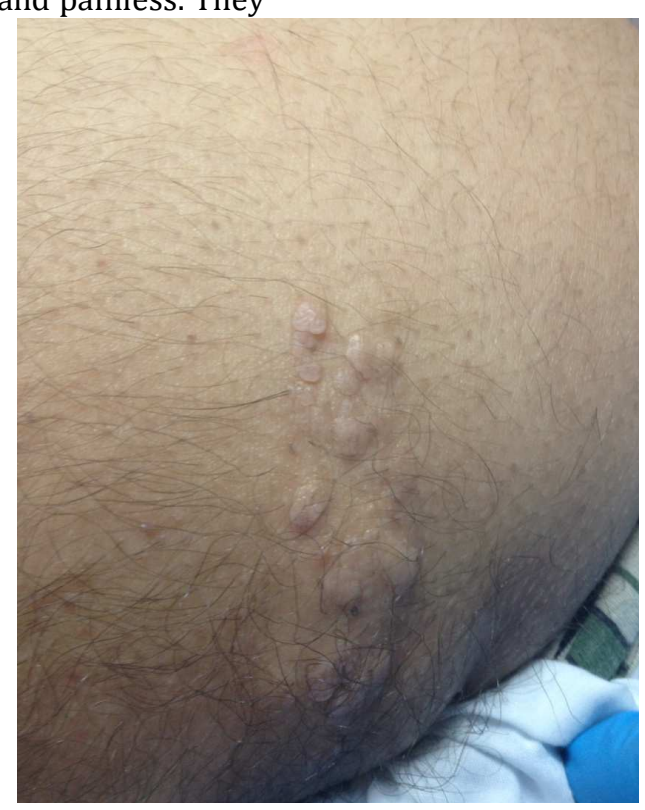

Figure 1 : Right lower buttock with segmental soft skin coloured lesions

The patient denied any history of trauma to the lesion site. The lesion was not however consisted of sessile skin coloured papules and nodules with smooth, shiny, and wrinkled surfaces, but they could not be separated out from the skin (figure 1). These papules and nodules were mimicking skin tags. Some were solitary and some were multiple coalesced symptomless unilateral plaques over the left buttock area (figure 1). They did not itch, hurt, or otherwise bother him; the patient was otherwise healthy. The main compliant of the patient was the mild discomfort when he had his trousers and underwear on. He also felt pressure whilst he sat on his buttock. He found the physical external appearance very unsightly and this affected his quality of life (QoL) and he wanted the lesions removed. There was no family history of similar kind of disease. growing but had remained unchanged over the last ten years. 
One nodule was excised for histological evaluation. Histopathological examination of the biopsy specimen revealed sections of the skin with fibro- collagenous tissue mixed with mature adipose tissues in the dermis, with overlying epidermis partly acanthotic at one edge. The dermal vessels were generally normal, with occasional dilated vessel. (The histopathological image cannot be provided due to the policy of the overseas lab and they state they are not equipped to do so).

Special fat staining was not conducted as the pathologist does not see it a necessity.

The diagnosis was made on the characteristic clinical aspects and confirmed by the typical histopathological findings of the mass.

\section{Discussion}

Naevus lipomatosis superficialis (NLS) is a relatively rare skin malformation disorder, characterized by ectopic adipose tissue in the dermis and the existing literature reported about 60 cases. NLS has neither sex nor familial trend predilection, and the first condition was described by Hoffman and Zurhelle in 1921 as Goucha et al (2011) declared. Few cases have been reported thereafter as Ghosh et al (2010), Bancalari et al (2011) stated. NLCS usually have an indolent and asymptomatic course, and can reach a large size if left untreated, and the largest size reported in the literature was $40 \mathrm{~cm} \mathrm{x28} \mathrm{cm} \mathrm{as} \mathrm{Goucha} \mathrm{et} \mathrm{al} \mathrm{(2011)}$ reported.

NLCS has been reported in association with some cutaneous disorders; such as angiokeratoma of Fordyce, scattered leukoderma, café-au-lait macules, and hemangioma as Goucha et al (2011) found. My case did not show any other associated skin abnormalities. The patient cannot recall precise duration of the lesion; however, the possible explanation could be that the lesion might be present at infancy and then grew during adolescence and noticed later, as Wollina 2013 said.
The pathogenesis of NLCS remains obscure. However, some cases present with ulceration due to ischemia in the dermal capillaries. This case looked to be the classic type lesions as the patient stated its existence since long time and did not bother him at all, just psychologically. The multiple lesions often have a marked predilection for the gluteal region as my case had revealed.

The postulation beyond this naevus type falls between either a deposition of adipose tissue secondary to degenerative changes in the dermal collagen and elastic tissue, or due to a true naevus resulting from the focal heterotropic development of adipose tissue. However, the real explanation is not yet settled as Sandipan et al (1994), Goucha et al (2011) and Rameshwar et al 2011 projected.

The most common sites affected are the pelvic, the lower back, ears, the gluteal region, and the thigh, including scalp cases documented as well. Occurrence over atypical sites like the nose, eyelid, calf and clitoris, vulva has also been documented in the literature as Khandpu et al (2009), Goucha et al (2011) had been found. Usually NLCS have an indolent and asymptomatic track as Goucha et al (2011) affirmed. It has been reported in some cases that NLC has grown near the ulnar nerve causing an entrapment with numbness. In these surgical, release was urgently needed as Süleyman and Hüsamettin, 2014 alleged. Sporadically, NLCS may ulcerate after external trauma or ischemia as yap 2009 stated. Moreover, in some cases, the surface of naevus could be studded with multiple open comedones, resulting in an annoying foul smell, as Bancalari et al and Goucha et al (2011) acknowledged.

The differential diagnoses consist of fibroma, neurofibroma, lymphangioma or hemangioma, fibroepithelial polyps, and lipomas. Frequently, NLS remains asymptomatic.

Lipoma is painless lump, slowly growing which is mobile with a soft consistency on examination. Fibroma can be painful with 
usually tendency for locally aggressive behavior. Hemangioma has a vascular component.

The diagnosis of NLCS was based on characteristic clinical aspects and confirmed by typical histopathological features from the skin biopsy taken, as Goucha et al (2011) reported.

Treatment is frequently not essential unless for cosmetic reasons. Surgical excision is curative, and recurrence is rare. Also cryotherapy was useful and yielded satisfactory results. Some cases were treated with $\mathrm{CO} 2$ successfully with no recurrence after one year follow up as Goucha et al (2011) pointed out.

My patient wanted to achieve the normal smooth contour of his right buttocks, as he felt the lesion had impacted on his psychology, social functioning and well being,

\section{References}

1. Bancalari E, Martínez-Sánchez D, Tardío JC. (2011) 'Naevus lipomatosis superficialis with a folliculosebaceous component: report of 2 cases', Patholog Res Int. 18; 2011:105973.

2. Buch AC, Panicker NK, Karve PP. (2005) 'Solitary naevus lipomatosis cutaneous superficialis', J Postgrad Med. 51(1):47-8.

3. Das JK, Sengupta S, Gangopadhyay AK. (2006) 'Naevus lipomatosis superficialis over neck, an atypical site', Indian J Dermatol Venereol Leprol.; 72(1):66-7.

4. Ghosh SK, Bandyopadhyay D, Jamadar NS. (2010) 'Naevus lipomatosis cutaneous superficialis: An unusual presentation', Dermatol Online J. Jul 15; 16 (7):12.

5. Goucha S, Khaled A, Zéglaoui F, Rammeh S, Zermani R, Fazaa B. (2011) 'Naevus lipomatosis cutaneous superficialis: Report of eight cases', Dermatol Ther (Heidelb). 1 (2): 25-30. and his main concern was mainly for aesthetic reasons.

The uniqueness of reporting this rare case is the patient's quality of life experiences. The physicians should consider patient complaints very careful as my patient wellbeing seemed to be affected overwhelmingly.

This case I am reporting is being reported for the rarity of the disorder. To the best of my knowledge, there are no systemic abnormalities or malignant transformation described with NLCS, and this is the first case reported from Libya.

Lastly, physicians should be conscious of this rare state to enable more conservative resection of the lesion if needed and thus less invasive reconstruction of the defect.

6. Khandpur S, Nagpal SA, Chandra S, Sharma VK, Kaushal S, Safaya R..(2009) 'Giant naevus lipomatosis cutaneous superficialis', Indian J Dermatol Venereol Leprol.; 75 (4):407-8.

7. Pujani M, Choudhury M, Garg T, Madan NK. (2014). 'Naevus lipomatosis superficialis: a rare cutaneous hamartoma'. Indian Dermatol Online J. 5(1):109-10.

8. Rameshwar G, Chikhalkar S, Mahajan S, Kharkar V, Khopkar U. (2011), 'Asymptomatic cerebriform papules and nodules over left buttock'. Indian Journal of Detmatology, Venerology and Leprology., vol 77 (2), p 254.

9. Sandipan D, Bhushan $\mathrm{K}$, Inderjeet $\mathrm{K}$. (1994) ' Naevus lipomatosis superficialis of Hoffman and Zurhelle', Indian J Dermatol Venereol Leprol, 60 , (1), 39-40.

10. Süleyman T, Hüsamettin T. (2014), 'Giant nevus lipomatosus cutaneous superficialis with intramuscular lipomatosis caused sciatic nerve compression'.J Cutan Med Surg. 18:1-2. 
11. Varadraj Vasant Pai, Kikkeri Narayanshetty Naveen, Sharatchandra Bhimrao Athanikar, Ravikala Rao, and Tukaram Sori. (2013) 'unusal presentation of naevus lipomatosis cutaneus superficialis' Indian J Dermatol. ; 58(6): 486487.

12. Wollina U. (2013), 'Photoletter to the editor
Nevus lipomatosus superficialis (HoffmannZurhelle). Three new cases including one with ulceration and one with ipsilateral gluteal hypertrophy'. J Dermatol Case Rep. 30; 7(2):71-3.

13. Yap FB. (2009), 'Naevus lipomatosis superficialis'. Singapore Med J., 50 (5):e161-2. 